\title{
Sensory experience modifies spontaneous state dynamics in a large-scale barrel cortical model
}

\author{
Elena Phoka • Mark Wildie . \\ Simon R Schultz • Mauricio Barahona
}

Received: date / Accepted: date

\begin{abstract}
While the neuronal activity of the cerebral cortex is strongly modulated by sensory inputs, the cortex also exhibits rich spontaneous dynamics. Experimental evidence suggests that sensory stimulation may shape the spontaneous activity of the cortex, which in turn can influence its responses to further external stimulation. However, we still do not understand how sensory stimuli affect the underlying neural circuitry. Here we study whether spike-timing-dependent plasticity (STDP) can mediate sensory-induced modifications in the spontaneous dynamics of a new large-scale model of layers II, III and IV of the rodent barrel cortex. A central feature of our model is its level of physiological detail, including the types of neurons present, the probabilities and delays of connections, and the STDP profiles at each excitatory synapse. We stimulated the neuronal network with a protocol of repeated sensory inputs, resembling those generated by the protraction-retraction motion of whiskers when rodents explore their environment, and studied the changes in network dynamics. By applying dimensionality reduction techniques to the synaptic weight space, we show that the trajectories converge to an initial spontaneous attractor state, which is modified by each repetition of the stimulus. This reverberation of the sensory experience induces long-term modifications in the synaptic weight space. The post-stimulus spontaneous state encodes a unique memory of the stimulus presented, since a different dynamical response is observed when the network is presented with shuffled stim-
\end{abstract}

E. Phoka

Department of Bioengineering \& Department of Mathematics, Imperial College London

E-mail: e.phoka07@imperial.ac.uk

M. Wildie

Department of Computing, Imperial College London

S.R. Schultz

Department of Bioengineering, Imperial College London

M. Barahona

Department of Mathematics, Imperial College London

E-mail: m.barahona@imperial.ac.uk 
uli. These results suggest that repeated exposure to the same sensory experience could induce long-term circuitry modifications via 'Hebbian' STDP plasticity.

Keywords barrel cortex · spontaneous dynamics · STDP · large-scale model

\section{Introduction}

A linear systems view has informed much of the way we think about sensory information processing in the cortex. Substantial advances were made based on the concept of receptive field to characterise the information processing operation performed by a neuron (Hartline 1938, Hubel \& Wiesel 1968), a concept that has been mathematically formalised with the development of linear-nonlinear cascade models of sensory information processing (Wu et al. 2006, Saleem et al. 2008, Pillow et al. 2008). However, cortical neurons are spontaneously active (Creutzfeldt et al. 1966, J. 1976, Kenet et al. 2003, Mao et al. 2001, Luczak et al. 2007, Sakata \& Harris 2009) due to both network and internally generated activity (Le Bon-Jego \& Yuste 2007) and the linear systems view of cortical information processing does not take into account a functional role for this spontaneous activity.

Spontaneous activity consumes a large fraction of the metabolic budget of the brain (Sokoloff et al. 1955, Raichle \& Mintun 2006). It would thus appear likely that spontaneous activity should have a functional role, which is yet to be understood. An increasing body of experimental evidence has led to the notion that neuronal responses are affected by the intrinsic spontaneous state of the system (Petersen et al. 2003, Tsodyks et al. 1999, Fiser et al. 2004, Ferezou et al. 2007). For example, cortical neuronal responses to repeated application of the same stimulus have a high degree of trial-to-trial variability (Arieli et al. 1996, Azouz \& Gray 1999, Fox et al. 2007), much of which is due to spontaneous membrane potential fluctuations (reflecting the spontaneous activity of the network incipient to a neuron). Furthermore, the spontaneous fluctuations in the membrane potential, the UP and DOWN states, were shown to affect the responsiveness of cortical neurons to whisker stimulation (Hasenstaub et al. 2007).

Complementary, as shown in voltage-sensitive dye imaging experiments, spatiotemporal patterns of activity do not merely reflect stochastic network fluctuations or internal noise sources, but can also be affected by the recent history of sensory experience (Kenet et al. 2003, Han et al. 2008, Yao et al. 2007). Similar results were obtained from human BOLD fMRI data, which showed a robust and specific learning-related modulation of spontaneous activity (Lewis et al. 2009). Along similar lines, Alenda et al. (2010) showed that an ongoing stimulus can modulate spontaneous membrane potential fluctuations throughout its duration. As a result, it was suggested that the cortex behaves somewhat like a central pattern generator, a system with rich spontaneous dynamics that is strongly modulated by, and responsive to, sensory inputs (Yuste et al. 2005).

While experimental evidence suggests that the spontaneous activity of the cortex may be shaped by sensory stimulation and can influence brain responses to external stimulation, we lack information on how sensory stimuli affect the underlying network circuitry. For example: Are the underlying spontaneous dynamics modified by sensory stimulation? Can repetitive sensory stimulation induce longlasting modifications in the neuronal connections? Could the neuronal network 
create new memory traces within the cortex by shifting the spontaneous state of the system? And if so, what mechanisms could account for such an effect?

Here we address these questions through computer simulations of the somatosensory (barrel) cortex. We specifically examine whether STDP can account for sensory-induced long-term modifications of the synaptic dynamics. The barrel cortex is a system whose well-defined anatomical structure has facilitated a thorough experimental examination of its inter- and intra-layer connectivity over the past years. Sensory inputs arrive in the barrel cortex via mainly the posterior thalamic nucleus (VPm) and the thalamic axons branch extensively within layer IV of the cortex (Cruikshank et al. 2009, Killackey \& Ebner 1973). More than $90 \%$ of connections of excitatory layer IV cells are made within layer IV and layers II/III (Lübke et al. 2000). We thus developed a large-scale model of layers II, III and IV of a barrel cortical column. The model is based on the physiological literature (e.g. Lefort et al. 2009), including both the numbers and proportions of neurons of each class, their connectivity probabilities and delays. Model parameters are tuned to achieve essential physiological features such as firing rates and stationarity. In addition, the synaptic strengths in the model evolve according to STDP rules with distinct dynamical profiles for the different synapses. Through the adoption of this mechanism for network dynamics, we investigate the role of Hebbian spike pairing in the modification of ongoing dynamics by sensory stimulation and how such a mechanism could underlie long-term plasticity effects driven by sensory inputs.

The evolution of the spontaneous state of the network was evaluated by considering a reduced dimensionality representation of the neuronal synaptic weight dynamics. When the system is stimulated with repeated instances of the same structured input, the pre-stimulus synaptic spontaneous attractor state shifts to new post-stimulus spontaneous synaptic state. The analysis of the dynamics in this reduced dimensionality space identifies a direction related to excitability (i.e., the short-term response to the stimulus) and a direction corresponding to network training (along which the long-term modification of the spontaneous state occurs). We show that the final post-stimulus attractor state exhibits distinct network synaptic activity trajectory in response to the structured input as compared to randomly shuffled inputs. This behaviour indicates that the final post-evoked spontaneous synaptic state of the network captures unique features of the presented sensory stimulus that affect the network response to sensory inputs.

\section{Model of layers II, III and IV of a barrel cortical column}

Our model is based on the known microanatomy of the barrel cortex and on information available in the literature about the types of neurons and their connectivity pattern. The model is divided into three laminae with different patterns of afferent, efferent and local connectivities. These laminae represent the supragranular layers (II and III) and layer IV of the barrel cortex. Electrophysiological recordings of layer II/III barrel neurons have identified three main cell types based on spike waveform and cell morphology: excitatory pyramidal ( $\mathrm{p}_{\mathrm{II} / \mathrm{III}}$ ) regular spiking (RS) neurons, and two main types of inhibitory neurons, the basket ( $\mathrm{b}_{\mathrm{II} / \mathrm{III}}$ ) fast spiking (FS) and the non-basket (nb $\mathrm{nI}_{\mathrm{II} / \mathrm{III}}$ ) low threshold spiking (LTS) interneurons (McCormick et al. 1985, Kawaguchi 1995, Gibson et al. 1999). In layer IV, 
the primary targets of thalamic afferents are the excitatory spiny stellates ( $\mathrm{SSIV}_{\mathrm{IV}}$ ), which are regular spiking neurons (Feldmeyer et al. 1999), and the inhibitory basket $\left(b_{I V}\right)$ fast spiking and non-basket $\left(\mathrm{nb}_{\mathrm{IV}}\right)$ low threshold spiking interneurons.

Our model contains 3717 spiking neurons (layer II: $546 \mathrm{p}_{\mathrm{II}}, 55 \mathrm{~b}_{\mathrm{II}}, 55 \mathrm{nb}$ II ; layer III: $1145 \mathrm{p}_{\text {III }}, 60 \mathrm{~b}_{\mathrm{III}}, 60 \mathrm{nb}_{\mathrm{III}}$; and layer IV: $1656 \mathrm{ss}_{\mathrm{IV}}, 70 \mathrm{~b}_{\mathrm{IV}}, 70 \mathrm{nb}$ IV (Lefort et al. 2009)). The proportion of excitatory to inhibitory neurons in layers II/III is approximately 9:1. The model includes intralaminar connections, vertical interlaminal connections, as well as thalamocortical connections for a total of 2,142,572 synaptic connections.

The neuronal connectivity pattern is shown in Figure 1A. All neuron types in the network are connected to all other neuronal types with probabilities given in Section 2.2, with the exception that supragranular interneurons do not connect to layer IV interneurons, because of weak experimental support. The spatial locations of the neurons were set at random and for vizualization purposes a $3 \mathrm{D}$ schematic is shown in Figure 1C. The delays of horizontal connections (in the same layer) for each neuron type were not significantly different between the different synapses, whereas the delays of the vertical connections were set to reflect the spatial distance of one layer from the other (see Section 2.2).

\subsection{Model dynamics}

The dynamics of each neuron in the network is modelled as described by Izhikevich \& Edelman (2008). Depending on the values of the parameters $a, b, c$ and $d$, this model reproduces the spiking dynamics of each of the three neuronal types: $\mathrm{RS}, \mathrm{FS}$ and LTS (Izhikevich \& Edelman 2008). The values of $a, b, c, d, v_{r}, v_{t}$, $v_{\text {peak }}, C$ and $k$ used in this study are shown in Table 1 . Heterogeneity in the model was introduced by jittering some of the parameters around a mean value as shown in Table 1 , where $r$ is a random deviate in the range $[-1,1]$. The deviations from the mean for parameters $v_{r}$ and $C$ were determined from experimental data that suggest a 7\% (Beierlein et al. 2003, Zhu \& Connors 1999, McCormick et al. 1985, Halabisky et al. 2006, Chagnac-Amitai et al. 1990) and 5\% (Gentet et al. 2000) magnitude standard deviation, respectively. For each neuron, $K$, its input current, $\mathrm{I}_{K}$ at each time step was modelled as the combined synaptic input: $\mathrm{I}_{K}=\sum_{L \in C} w_{K L}$, where $w_{K L}$ is the weight of the synapse from neuron $K$ to neuron $L$ and $C$ is the set of neurons with synapses terminating at $K$. The weight between neurons $K$ and $L$ changes according to the spike-pairing rules described in Section 2.3.

\subsection{Connection probabilities and delays}

The probabilities of synaptic connections between any two neurons, as well as the propagation delays for signal transmission between neurons, were derived from the literature. The values used for the neuronal connectivity probabilities are listed in Table 2. Where available, the table entries were taken from the literature and as above, the entries with asterisks denote values taken from Lefort et al. (2009).

Previous studies have investigated the relationship between the probability of connections and the distance between neurons. Specifically, Holmgren et al. (2003) 
Table 1 Model parameters for each neuron type according to Izhikevich \& Edelman (2008). The entries with asterisks are taken from Lefort et al. (2009).

\begin{tabular}{|c|c|c|c|c|c|c|c|c|c|c|}
\hline Morphology & Cell Type & $a$ & $b$ & $c$ & $d$ & $v_{r}$ & $v_{t}$ & $v_{\text {peak }}$ & $C$ & $k$ \\
\hline $\mathrm{p}_{\text {II }}$ & $\mathrm{RS}$ & 0.01 & 5 & -60 & $300+100 r$ & $-72+0.3 r^{*}$ & -50 & 50 & $100+5 r$ & 3 \\
\hline $\mathrm{b}_{\mathrm{II}}$ & FS & 0.15 & 8 & -55 & $200+100 \mathrm{r}$ & $-55+3.9 \mathrm{r}$ & -40 & 25 & $20+\mathrm{r}$ & 1 \\
\hline $\mathrm{nb}_{\text {II }}$ & LTS & 0.03 & 8 & -50 & $14+2 \mathrm{r}$ & $-56+3.9 r$ & -42 & 40 & $100+5 r$ & 1 \\
\hline $\mathrm{p}_{\text {III }}$ & $\mathrm{RS}$ & 0.01 & 5 & -60 & $300+100 r$ & $-71.4+0.4 r^{*}$ & -50 & 50 & $100+5 \mathrm{r}$ & 3 \\
\hline $\mathrm{b}_{\text {III }}$ & FS & 0.15 & 8 & -55 & $200+100 r$ & $-55+3.9 \mathrm{r}$ & -40 & 25 & $20+r$ & 1 \\
\hline $\mathrm{nb}_{\mathrm{III}}$ & LTS & 0.03 & 8 & -50 & $18+2 \mathrm{r}$ & $-56+3.9 \mathrm{r}$ & -42 & 40 & $100+5 r$ & 1 \\
\hline $\operatorname{ss}_{I V}$ & $\mathrm{RS}$ & 0.01 & 5 & -60 & $300+100 r$ & $-66+0.3 r^{*}$ & -50 & 50 & $100+5 r$ & 3 \\
\hline $\mathrm{b}_{\mathrm{IV}}$ & FS & 0.15 & 8 & -55 & $200+100 r$ & $-55+3.9 \mathrm{r}$ & -40 & 25 & $20+\mathrm{r}$ & 1 \\
\hline$n b_{I V}$ & LTS & 0.03 & 8 & -50 & $18+2 \mathrm{r}$ & $-56+3.9 \mathrm{r}$ & -42 & 40 & $100+5 \mathrm{r}$ & 1 \\
\hline
\end{tabular}

Table 2 Probabilities of connections between neuron types (in percentage).

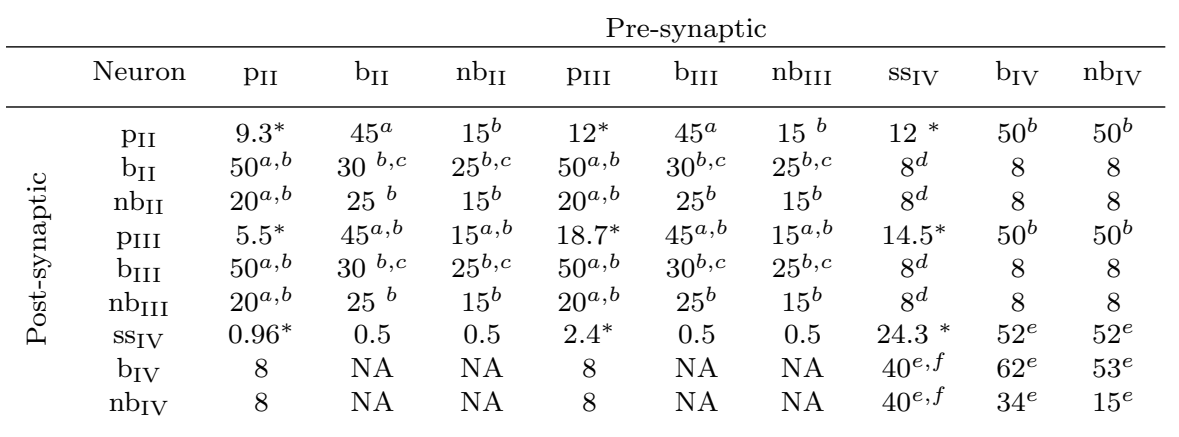

$\bar{a}$ Holmgren et al. (2003) (rat), ${ }^{b}$ Thomson et al. (2002) (rat), ${ }^{c}$ Galarreta et al. (2008) (mouse), ${ }^{d}$ Helmstaedter et al. (2008) (rat), ${ }^{e}$ Gibson et al. (1999) (rat), ${ }^{f}$ Lübke et al. (2000) (rat), ${ }^{*}$ Lefort et al. (2009) (mouse)

reported a decrease from $9 \%$ probability of two cells within $\pm 25 \mu \mathrm{m}$ of each other being connected, to $1 \%$ at distances $>100 \mu \mathrm{m}$ for $\mathrm{p}_{\mathrm{II} / \mathrm{III}}-\mathrm{P}_{\mathrm{II} / \mathrm{III}}$ synapses in visual and somatosensory cortex. However, Song et al. (2005) reported that the probability of connection does not depend systematically on the distance in the same neuronal type in the visual cortex. In support of the latter, Lefort et al. (2009) found no significant drop in connectivity over the distances explored in a single mouse barrel. As a result, there is not sufficient evidence to suggest a significant decrease in connection probability with distance within the same barrel and, consequently, we have not incorporated such dependence in our model.

The delays of signal propagation for connections between and within the different cell types were set according to experimental findings, as listed in Table 3. As above, $r$ is a random deviate in the range [-1 1$]$. Where available, the table entries were taken from the literature and the rest of the entries are estimates (based on known values from the literature within or between the layers). There is no evidence to suggest an increase of synaptic delays with the distance between neurons within a layer of barrel cortex. For example, Mason et al. (1991) found no correlation between latency and the separation of recording sites, ranging from 50 to $350 \mu \mathrm{m}$, in visual cortex using in vitro electrophysiology. Thus, we have set the delays between neurons confined within a layer to be determined by the mean and 


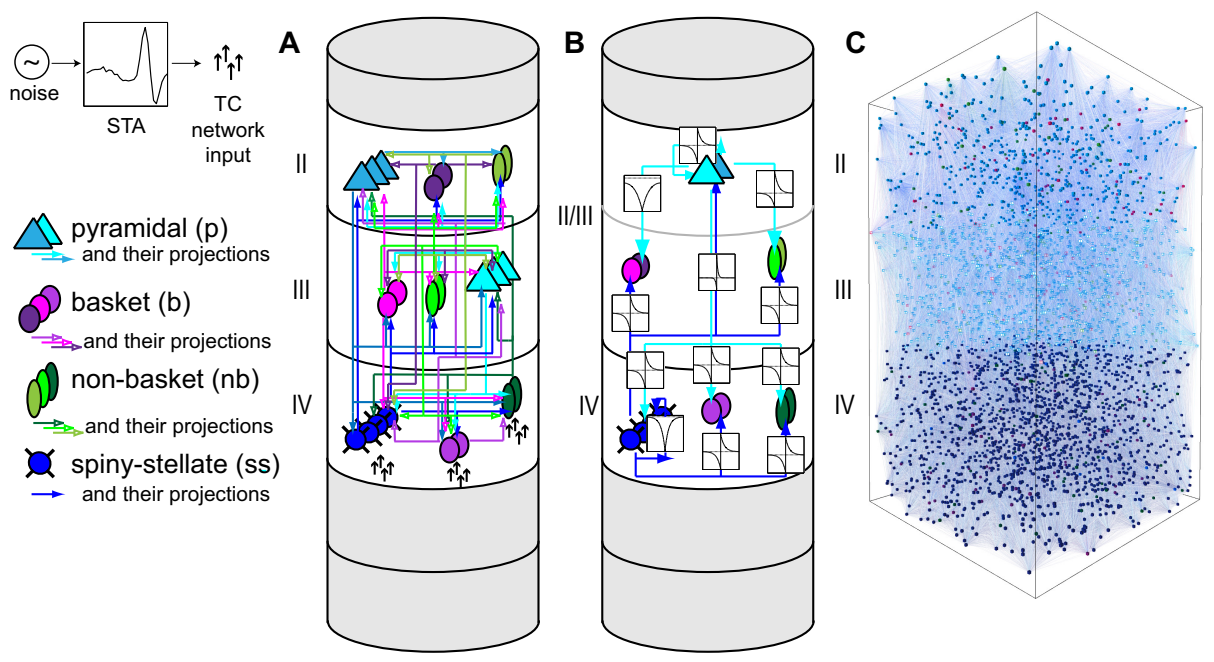

Fig. 1 Representation of the model of barrel cortical circuitry of layers II, III and IV. (a) All neuronal types in the network are connected to all other neuronal types, except supragranular interneurons to layer IV interneurons. The model contains 3717 spiking neurons whose synaptic connections, along with the delays between neurons, were based on the physiological literature. It includes 6 neuronal types: in layers II and III, excitatory pyramidal ( $\left.\mathrm{p}_{\mathrm{II} / \mathrm{III}}\right)$ and inhibitory basket $\left(\mathrm{b}_{\mathrm{II} / \mathrm{III}}\right)$ and non-basket $\left(\mathrm{nb}_{\mathrm{II} / \mathrm{II}}\right)$ interneurons; in layer IV, spiny stellates $\left(\mathrm{ss}_{\mathrm{IV}}\right)$ and inhibitory basket ( $\left.\mathrm{b}_{\mathrm{IV}}\right)$ and non-basket $\left(\mathrm{nb}_{\mathrm{IV}}\right)$. The thalamocortical (TC) spiketrain inputs are calculated by convolving thalamic STAs with external stimuli (top inset) (b) The different STDP dynamic profiles for the excitatory synapses are shown in this simplified wiring diagram. The neurons in layers II and III share the same STDP profiles-hence depicted as one layer (layer II/III) in this diagram. Most synaptic modifications follow a symmetric plasticity rule (e.g., the $\mathrm{p}_{\mathrm{II} / \mathrm{III}}-\mathrm{p}_{\mathrm{II} / \mathrm{III}}$ synapses). The plasticity rules were modified when additional information was available from the current literature (e.g., the $\mathrm{p}_{\mathrm{IL} / \amalg}-\mathrm{nb}_{\amalg / \amalg}$ synapses). (c) A visualisation of the full network of 3717 neurons and 2.2 million synapses in layers II, III and IV. The depicted 3D structure of the network is for demonstration purposes only. A movie animation of the dynamics in this network can be found in the Supplementary Material.

standard deviation as shown in Table 3 and the delays between far layers were set to be larger than the delays within or to neighbouring layers. The laminar structure of the model is achieved by adjusting the synaptic delays between layers to be higher than the intralaminar delays since larger delays are expected between rather than within layers.

\subsection{Spike-timing dependent plasticity}

The dynamics of the excitatory synapses in our model evolve under STDP, i.e., long-term synaptic modification induced by pairing of pre- and postsynaptic action potentials as a function of their relative timing. We take into account the fact that different synapses display different profiles of STDP. We did not include STDP in the inhibitory synapses due to insufficient experimental support. The STDP introduced in the model is based on a NonLinear Temporal Asymmetric Hebbian (NLTAH) learning rule, which allows continuous interpolation between additive and mulitplicative models, as proposed by Gütig et al. (2003). 


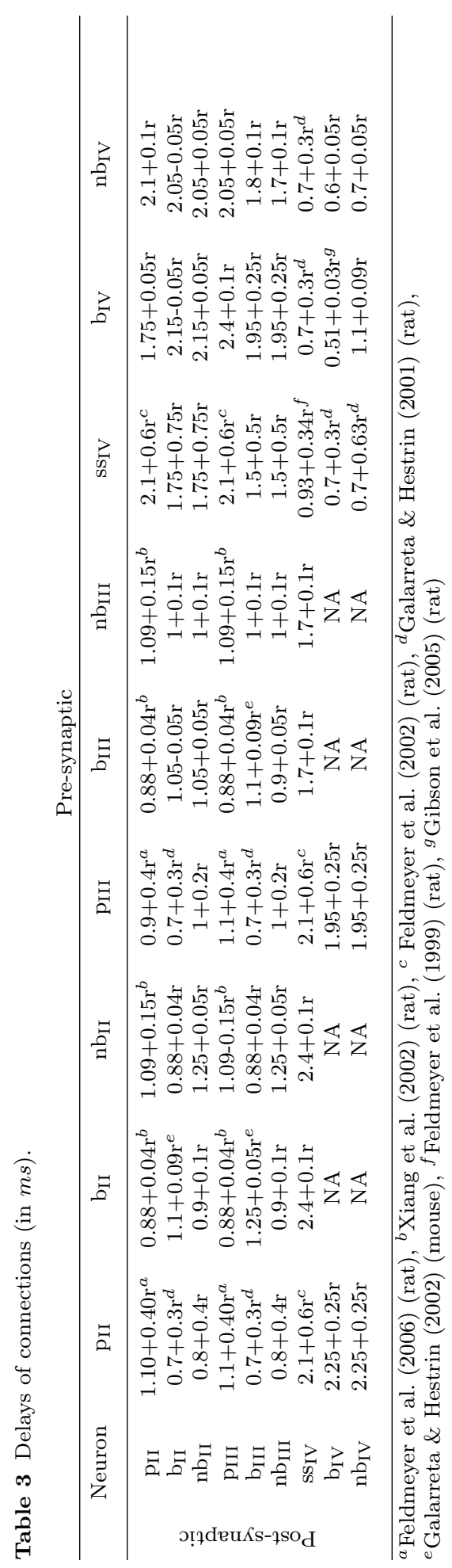


Table 4 STDP parameters.

\begin{tabular}{|c|c|c|c|c|c|c|}
\hline Synapse & $\lambda$ & $\alpha$ & $\mu_{+}$ & $\mu_{-}$ & $\tau_{+}(m s)$ & $\tau_{-}(m s)$ \\
\hline Default ${ }^{a}$ & 0.06 & 1.0 & 0.5 & 0.5 & 20.0 & 20.0 \\
\hline $\mathrm{p}_{\mathrm{II} / \mathrm{III}}-\mathrm{b}_{\mathrm{II} / \mathrm{III}}^{b}$ & 0.001 & 1.0 & 0.5 & 0.5 & 39.1 & 39.9 \\
\hline $\mathrm{p}_{\mathrm{II} / \mathrm{III}}{ }^{-n b_{\mathrm{II} / \mathrm{III}}}{ }^{b}$ & 0.25 & 0.6 & 0.5 & 0.5 & 20.0 & 20.0 \\
\hline $\mathrm{p}_{\mathrm{II} / \mathrm{III}} / \mathrm{ss}_{\mathrm{IV}}-\mathrm{b}_{\mathrm{IV}}$ & 0.03 & 1.0 & 0.5 & 0.5 & 20.0 & 20.0 \\
\hline $\mathrm{SS}_{\mathrm{IV}}-\mathrm{p}_{\mathrm{II} / \mathrm{III}}{ }^{c}$ & 0.13 & 0.5 & 0.5 & 0.5 & 6.0 & 40.0 \\
\hline $\mathrm{sS}_{\mathrm{IV}}-\mathrm{b}_{\mathrm{II} / \mathrm{III}}$ & 0.02 & 1.0 & 0.5 & 0.5 & 20.0 & 20.0 \\
\hline $\mathrm{SS}_{I V}-\mathrm{SS}_{\mathrm{IV}} d$ & 0.0011 & 1.0 & 0.5 & 0.5 & 20.0 & 20.0 \\
\hline
\end{tabular}

${ }^{a}$ Markram et al. (1997) (rat), ${ }^{b} \mathrm{Lu}$ et al. (2007) (rat)

${ }^{c}$ Feldman (2000) (rat), ${ }^{d}$ Egger et al. (1999) (rat)

Unless otherwise stated, the synaptic modifications follow a symmetric plasticity rule with a classic anti-symmetrical exponential profile, which we denote as 'default STDP'. However, experimental evidence exists in support of different STDP profiles in some synapses. For example, the $\mathrm{p}_{\mathrm{II} / \mathrm{III}}-\mathrm{b}_{\mathrm{II} / \mathrm{III}}$ and $\mathrm{SS}_{\mathrm{IV}}{ }^{-} \mathrm{SS}_{\mathrm{IV}}$, follow a symmetric profile as depicted in Figure 1B. The parameters for the default STDP as well as for the synapses which follow different STDP plasticity rules are listed in Table $4 . \lambda$ denotes the learning rate, $\alpha>0$ denotes a possible asymmetry between potentiation and depression, $\mu$ determines the range of the boundary effects on the changes of the synaptic weights and the time constant, $\tau$, determines the temporal extent of the learning window (Gütig et al. 2003). Note that for the $\mathrm{SSIV}_{\mathrm{IV}}^{-\mathrm{SS} I V}$ and $\mathrm{p}_{\mathrm{II} / \mathrm{III}}-\mathrm{b}_{\mathrm{II} / \mathrm{III}} \mathrm{STDP}$ rules, $\lambda$ is set to a value which is two or three orders of magnitude lower than the rest of the STDP rules. This is because the weights of these synapses are depressed independently of the presynaptic/postsynaptic spike-time order (Egger et al. 1999, Lu et al. 2007). In addition, for the $\mathrm{SS}_{\mathrm{IV}}-\mathrm{b}_{\mathrm{IV}} / \mathrm{b}_{\mathrm{II} / \mathrm{III}}$ and $\mathrm{p}_{\mathrm{II} / \mathrm{III}}$ - $\mathrm{b}_{\mathrm{IV}}$ STDP rules, $\lambda$ was set to lower values than those in the default STDP, in order to prevent rapid decay of these connections. The corresponding profiles for each of the synapses are illustrated in Figure 1B.

\subsection{Synaptic release}

Two problems frequently encountered with large-scale neural simulations are instability and non-stationarity. In particular, spontaneous activity is often found to die out over time (Izhikevich \& Edelman 2008) - i.e. it is unstable. One physiologically inspired solution to this is to introduce spontaneous synaptic release (del Castillo \& Katz 1954). In order to maintain the activity of the neurons of the network at constant levels, miniature (low-amplitude) excitatory potentials (synaptic release) were introduced at all synapses and modelled as Poisson noise. The amount of noise introduced was synapse-specific and the values are shown in Table 5. The amount of noise together with the synaptic weight values were used to adjust the neuronal firing rates and to prevent the spontaneous activity from dying off. 
Table 5 Synaptic release for each type of synapse $(H z)$.

\begin{tabular}{|c|c|c|c|c|c|c|c|c|c|c|}
\hline & & & & & & e-syr & ptic & & & \\
\hline & Neuron & $\mathrm{p}_{I I}$ & $\mathrm{~b}_{\text {II }}$ & $\mathrm{nb}_{\mathrm{II}}$ & $\mathrm{p}_{\text {III }}$ & $\mathrm{b}_{\text {III }}$ & $\mathrm{nb}_{\text {III }}$ & $\mathrm{SS}_{\mathrm{IV}}$ & $\mathrm{b}_{\text {IV }}$ & $\mathrm{nb}_{\text {IV }}$ \\
\hline & $\mathrm{p}_{\text {II }}$ & 470 & 230 & 230 & 470 & 230 & 165 & 250 & 230 & 230 \\
\hline & $\mathrm{b}_{\text {II }}$ & 140 & 240 & 240 & 140 & 240 & 240 & 200 & 240 & 240 \\
\hline 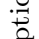 & $\mathrm{nb}_{\text {II }}$ & 40 & 40 & 40 & 35 & 40 & 40 & 25 & 40 & 40 \\
\hline$\underset{\sigma}{\sigma}$ & $\mathrm{p}_{\text {III }}$ & 410 & 230 & 230 & 410 & 230 & 165 & 250 & 230 & 230 \\
\hline $\overrightarrow{\sigma_{2}}$ & $\mathrm{~b}_{\text {III }}$ & 120 & 210 & 210 & 120 & 210 & 210 & 170 & 210 & 210 \\
\hline$\frac{1}{10}$ & nb & 40 & 40 & 40 & 25 & 40 & 40 & 25 & 40 & 40 \\
\hline 0 & SSIV & 200 & 115 & 115 & 200 & 115 & 115 & 210 & 115 & 115 \\
\hline & $\mathrm{b}_{\mathrm{IV}}$ & 0.2 & NA & NA & 0.2 & NA & NA & 0.2 & 380 & 380 \\
\hline & $\mathrm{nb}_{\mathrm{IV}}$ & 20 & NA & NA & 20 & NA & NA & 20 & 30 & 115 \\
\hline
\end{tabular}

Table 6 Averaged weights reached after equilibration at 30 seconds of simulated realtime.

\begin{tabular}{|c|c|c|c|c|c|c|c|c|c|c|}
\hline & & & & & & re-syı & tic & & & \\
\hline & Neuron & $\mathrm{p}_{I I}$ & $\mathrm{~b}_{\mathrm{II}}$ & $\mathrm{nb}_{\mathrm{II}}$ & pIII & $\mathrm{b}_{\text {III }}$ & nb III & $\mathrm{s}_{\mathrm{IV}}$ & $\mathrm{b}_{\text {IV }}$ & $\mathrm{nb}_{\mathrm{IV}}$ \\
\hline & $\mathrm{p}_{\text {II }}$ & 23.2 & -70 & -53 & 21.8 & -70 & -52.5 & 11.9 & -66 & -65.7 \\
\hline & $\mathrm{b}_{\mathrm{II}}$ & 0.4 & -8.8 & -53 & 0.42 & -9.6 & -52.5 & 25 & -8.4 & -10.5 \\
\hline$\because$ & $\mathrm{nb}_{\mathrm{II}}$ & 68.4 & -175 & -263 & 67.6 & -175 & -262.5 & 47.8 & -42 & -42 \\
\hline త్రి & $\mathrm{p}_{\text {III }}$ & 22.8 & -70 & -53 & 21.9 & -70 & -52.5 & 9.7 & -65.7 & -65.7 \\
\hline$\sum_{\hat{\Omega}}^{\tilde{N}}$ & $\mathrm{~b}_{\mathrm{III}}$ & 0.89 & -8.8 & -70 & 0.9 & -10.5 & -70 & 27.8 & -8.4 & -12.6 \\
\hline$\frac{1}{6}$ & $\mathrm{nb}_{\text {III }}$ & 72.2 & -175 & -263 & 71.9 & -175 & -262.5 & 49.2 & -63 & -63 \\
\hline 2 & SIV & 54.8 & -158 & -158 & 54.5 & -158 & -158 & 1.4 & -16.8 & -16.8 \\
\hline & $\mathrm{b}_{\text {IV }}$ & 60.3 & NA & NA & 59.6 & NA & NA & 53.4 & -2.1 & -2.1 \\
\hline & $n b_{I V}$ & 50.6 & NA & NA & 49.1 & NA & NA & 47.3 & -126 & -126 \\
\hline
\end{tabular}

2.5 Connection strengths and neuronal firing rates at stationarity

The weights of the synapses, in combination with the synaptic release, were adjusted to produce firing rates similar to those recorded in vivo (Zhu \& Connors 1999, Margrie et al. 2002, Armstrong-James \& Fox 1987) as well as maintaining the stability of the spontaneous activity of the network. The initial excitatory weights were allowed to evolve with time (governed by the STDP rules described above) until the dynamics had equilibrated, i.e., the initial transient of the dynamics had died off (see Figure 2A). The value of the weights reached after 30 seconds of simulated realtime for all the neuronal types are summarised in Table 6. Similarly, the average firing rates of all neuronal types reached after 30 seconds of simulated realtime are summarised in Table 7 .

\subsection{Sensory stimulation}

We present the network with a repeated structured stimulus that is built to resemble the sensory input that results from the motion of the vibrissae when swept across an object of detailed texture. During active exploration of their environment, rodents rhythmically sweep their whiskers over objects at a frequency of 5-25 Hz (Carvell \& Simons 1990, Lee \& Woolsey 1975, Jin et al. 2004, Brecht et al. 2006). In order to mimic the protraction/retraction motion of the whiskers 
Table 7 Firing rates reached after equilibration (after 30 seconds of simulated realtime).

\begin{tabular}{cc}
\hline Neuron & Firing rate $(\mathrm{Hz})$ \\
\hline $\mathrm{p}_{\mathrm{II}}$ & 1.5 \\
$\mathrm{~b}_{\text {II }}$ & 29.3 \\
$\mathrm{nb}$ & 62.5 \\
$\mathrm{p}_{\mathrm{III}}$ & 8.7 \\
$\mathrm{~b}_{\mathrm{III}}$ & 24.2 \\
$\mathrm{nb}$ & 44.1 \\
$\mathrm{SS}$ & 0.4 \\
$\mathrm{~b}$ & 64.6 \\
$\mathrm{n} \mathrm{b}_{\mathrm{IV}}$ & 67.7 \\
\hline
\end{tabular}

during an exploration event, we consider an external stimulus that consists of blocks with 10 repetitions per second of the same pattern of white noise appended to its mirror image (Figure 2B lower panel, light and dark grey). The 'back-andforth' patterns are repeated 10 times per second to mimic the $10 \mathrm{~Hz}$ vibrations of the whiskers during exploration. The duration of each stimulus block is 5 seconds and the stimulation protocol consisted in the repetition of this 5-minute block input 4 times (Figure 2B).

The sensory signals from the vibrissae reach the barrel cortex via mainly the ventroposterior medial nucleus (VPm) of the thalamus (Montemuro et al. 2007). The thalamic afferents project to layer IV neurons: onto spiny stellate neurons with $37 \%$ probability and onto inhibitory interneurons with $63 \%$ probability (Bruno \& Simons 2002) (see Figure 1A diagram). Similarly to Kyriazi \& Simons (1993), we modelled thalamocortical (TC) synaptic inputs using data recorded from the thalamus while stimulating the whiskers (Figure 1A). We specifically used thalamic transfer functions, spike-triggered averages (STAs), calculated by recording VPm responses while stimulating the vibrissae with approximately white noise. 14 different STAs were kindly provided to us by R. Petersen (Montemuro et al. 2007). For an example of an STA, see Figure 1A, top inset. The delays of TC projections were set to $1 \mathrm{~ms}$ and these synapses were not plastic.

The thalamic component in our model, was present both during spontaneous activity and sensory experience. In order to produce the thalamic spiketrains during sensory experience, each of 14 STAs were convolved with the external stimulus described in the preceding paragraph and a non-linear input-output function was used to produce an inhomogeneous Poisson spike train of time-averaged rate $\sim 30$ $H z$. To model the thalamic component of ongoing spontaneous activity, the STA was convolved with a low amplitude white gaussian noise (unrelated to the sensory stimulus) and the modeled spike train was chosen to fire at approximately $5 \mathrm{~Hz}$. The choice of white noise stimulus is justified by the fact that white noise has a flat power spectrum and, hence, neuron responses are sampled equally over a wide range of stimulus frequencies with equal weight (Marmarelis \& Naka 1972). The resulting spike-trains were used as synaptic input to layer IV neurons. The convolution and the non-linear input-output procedure was repeated for each of the 4 blocks, to ensure the variability in the thalamic spiketrains fed into the cortex, which is likely to occur with different whisks on the same object. Although the 5 -second stimulus was perfectly repeated 4 times, the thalamic spiketrains fed 
into the cortex were not perfectly repeated. Therefore, each block of 50 repetitions approximates one exposure to a detailed object.

The shuffled stimuli were produced by randomly permuting the 5 -second stimulus and thus no repetitions are expected in their structure. Each of the shuffled stimuli were convolved with each of the 14 STAs and passed through a non-linear input-output function.

\section{Computer Implementation}

The network was simulated using the Neural Simulation Tool (NEST) (Gewaltig \& Diesmann 2007) on the Imperial College High Performance Computing (HPC) system. The number of dynamical variables implemented in the model was approximately 1.8 million. Several modifications were implemented on the NEST simulation environment, including: the modification of the Message Passing Interface (MPI) implementation of NEST to include the Izhikevich neuron model; the generation of spontaneous synaptic activity (synaptic release); saving and restoring of model state for multi-part simulation; and efficient recording of data to allow the state of every neuron and synapse in the model to be sampled at $100 \mathrm{~ms}$ intervals. A simulation of 120 seconds of the model required approximately 442 minutes when run over 12 processors of the HPC system, generating 35 gigabytes of data. Processing of the resulting data set was carried out with a custom library of $\mathrm{C}$ and Python scripts, before final analysis in MATLAB (The MathWorks Inc.). Post-processing of the data corresponding to a 120 second simulation required an additional 1980 minutes of computation. Additional visualization of the results was carried out using the Java Processing visualization library.

\section{Analysis Methodology}

\subsection{Multidimensional Scaling}

The state of the system is defined by a high-dimensional vector containing the value of all synaptic weights at each point in time. In order to study dynamic changes of the network due to sensory stimulation, we use Multidimensional Scaling (MDS) to find a reduced dimensionality representation that encapsulates the essential intrinsic properties of the data (Kruskal 1964). MDS reduces the dimensionality of the problem following a geometric heuristic: it considers $\mathrm{X}$ objects in the high-dimensional space and projects them down to a reduced dimensional space attempting to preserve the inter-point geometric distances and, hence, introducing minimal distortion. Given a $d_{i j}$ distance between the original measurements $i$ and $j$, MDS finds a geometric representation $\hat{d}_{i j}$ in a lower-dimensional space by minimizing a cost function defined, for instance, as :

$$
\text { stress }=\sqrt{\frac{\sum_{i<j}\left(d_{i j}-\hat{d}_{i j}\right)^{2}}{\sum_{i<j} d_{i j}^{2}}}
$$


(Kruskal 1964) or

$$
\mathrm{s}-\text { stress }=\sqrt{\frac{\sum_{i<j}\left(d_{i j}^{2}-\hat{d}_{i j}^{2}\right)^{2}}{\sum_{i<j} d_{i j}^{4}}}
$$

(Takane et al. 1977).

Inter-point distances can be seen to correspond to dissimilarities between the original X objects and MDS works by assuming that dissimilarity between data is monotonically related to a notion of distance (Kruskal 1964). As a result, similar measurements in the original data remain close in the reduced representation and dissimilar measurements are kept apart in the geometric representation. There are also non-metric versions of MDS, in which the dissimilarities of the data are based on the rank order of entries in the data matrix and a configuration of points is found such that their distances reflect as closely as possible the rank order of the data.

In order to identify the correct dimension to represent the data accurately, the stress should be calculated for a range of increasing dimensions. If an 'elbow' in the stress-dimension plot appears at a dimension $\mathrm{D}$, and the value of the stress is low, this provides an indication for an appropriate reduced dimensionality for the data. MDS has been previously used to vizualise high-dimensional data in a variety of disciplines, including neuroscience (Kruskal \& Wish 1978, Cox \& Cox 2001, Luczak et al. 2009).

Our original synaptic weight vector consists of approximately 1,700,000 excitatory synapses evolving over 120,000 time points $(m s)$. Firstly, we reduced the dimensions of the vector from $1,700,000$ to 2983 by coarse-graining (block-averaging over every 600 synapses but ensuring that we preserved the original proportion of neuronal types). In addition, we subsampled every $100 \mathrm{~ms}$, thus reducing the time dimension to 1200 . Note that in our analysis we used only time points after stationarity was reached, i.e., above 30,000 $\mathrm{ms}$. Hence, the resulting coarse-grained matrix used for the analysis of the weight dynamics has dimensions [WxT]: [2983x901]. In order to study the time evolution of the neuronal ensemble, we used MDS to reduce this high-dimensional $[\mathrm{W} \times \mathrm{T}]$ matrix of weights to a $[\mathrm{D} \times \mathrm{T}]$ matrix, where $\mathrm{D}$ is the reduced dimension. We used the non-metric s-stress given in Eq. (2) for minimization of the error between the original and the projected space distances.

\subsection{Gaussian Process Regression}

To test whether the post-stimulus state attractor of the network, which emerges after several repetitions of the same block sensory stimulus, is unique to the stimulus presented, we tested the response of the network to shuffled stimuli. We presented the network with $N$ instances of shuffled stimuli and we evaluated whether the trajectory induced by the structured input can be obtained by chance, i.e., whether the structured response falls into the distribution of trajectories induced by the shuffled stimuli. To do so, we represented the trajectories following the shuffled stimuli by a multivariate Gaussian process and tested whether the trajectory induced by the structured input can be predicted by its distribution.

The set of shuffled trajectories consists of $N$ trajectories of length $u, \mathbf{s}_{\mathbf{i}}=$ $\left[s_{1}, s_{2}, \ldots, s_{u}\right]$ (dependent variables) sampled at $\mathbf{t}_{\mathbf{i}}=\left[t_{1}, t_{2}, \ldots, t_{u}\right]$ (independent variables) where $i=1, \ldots, N$. All the trajectories are appended to form a $N u \times 1$ 
vector $\mathbf{s}=\left[\mathbf{s}_{1} \ldots \mathbf{s}_{N}\right]$ and, similarly, all the observation times are compiled into the $N u \times 1$ vector $\mathbf{t}=\left[\mathbf{t}_{1} \ldots \mathbf{t}_{N}\right]$. Our training set is thus given by the pair of $N u \times 1$ vectors: $(\mathbf{t}, \mathbf{s})$. This set will be compared to a 'test' trajectory $\left(\mathbf{t}^{*}, \mathbf{s}^{*}\right)$, where both vectors are $u \times 1$.

The function that maps $\mathbf{t}$ into $\mathbf{s}$ can be estimated through the use of nonparametric probabilistic Gaussian Process Regression (Rasmussen \& Nickisch 2010). A Gaussian process (GP) is a collection of random variables with a joint multivariate Gaussian distribution defined by a mean and the covariance function (or kernel) $k\left(t_{p}, t_{q}\right)$. A common choice for $k\left(t_{p}, t_{q}\right)$ is the squared exponential covariance function (Rasmussen \& Nickisch 2010):

$$
k\left(t_{p}, t_{q}\right)=\sigma_{f}^{2} \exp \left[-\frac{1}{2 l^{2}}\left(t_{p}-t_{q}\right)^{2}\right]+\sigma_{n}^{2} \delta_{p q}
$$

where $\delta_{p q}$ is a Kronecker delta. The hyperparameters of the kernel function $\Theta=$ $\left[\sigma_{f}, l, \sigma_{n}\right]$ are estimated as those that maximize the marginal likelihood of the data.

Once the hyperparameters are estimated, we can obtain $G P_{\text {shuffled }}$, the Gaussian process that describes the shuffled data, which is given by the following multivariate Gaussian (Rasmussen \& Nickisch 2010):

$$
\begin{aligned}
G P_{\text {shuffled }} & \sim \mathcal{N}(\boldsymbol{\mu}, \boldsymbol{\Sigma}) \\
\text { with } \boldsymbol{\mu} & =K\left(\mathbf{t}^{*}, \mathbf{t}\right) K(\mathbf{t}, \mathbf{t})^{-1} \mathbf{s} \\
\text { and } \boldsymbol{\Sigma} & =K\left(\mathbf{t}^{*}, \mathbf{t}^{*}\right)-K\left(\mathbf{t}^{*}, \mathbf{t}\right) K(\mathbf{t}, \mathbf{t})^{-1} K\left(\mathbf{t}, \mathbf{t}^{*}\right) .
\end{aligned}
$$

Here $K\left(\mathbf{t}^{*}, \mathbf{t}\right)$ denotes the $u \times N u$ covariance matrix evaluated at all pairs of test and training time points and, similarly, the other covariance matrices are: $K(\mathbf{t}, \mathbf{t})$ (with dimensions $N u \times N u), K\left(\mathbf{t}^{*}, \mathbf{t}^{*}\right.$ ) (with dimensions $u \times u$ ) and $K\left(\mathbf{t}, \mathbf{t}^{*}\right)$ (with dimensions $N u \times u$ ). This multivariate Gaussian is the predictive distribution $p\left(\mathbf{s}^{*} \mid \mathbf{t}^{*}, \mathbf{s}\right)$, i.e., the distribution of observing the test-induced trajectory $\mathbf{s}^{*}$ at times $\mathbf{t}^{*}$ given the observed shuffled data set $\mathbf{s}$. The Gaussian is fully described by its time-varying mean $\boldsymbol{\mu}$ and covariance $\boldsymbol{\Sigma}$.

We estimated the $G P_{\text {shuffled }}$ using 19 shuffled trajectories and used a $20^{\text {th }}$ shuffled trajectory to cross-validate the predictive performance of the model and to compare it to a trajectory from the structured input. This allowed us to test whether the trajectory induced by the structured input belongs in $G P_{\text {shuffled }}$. The analysis was performed with the Gaussian Processes for Machine Learning (GPML) Toolbox (Rasmussen \& Nickisch 2010) and we used the squared exponential covariance function given in Eq. (3).

\section{Results}

We developed a model of the supragranular layers (II and III) and layer IV of a cortical column in the rodent barrel cortex, based on available information from the literature as detailed in Section 2. In summary, the model contains 3717 spiking neurons modeled as Izhikevich spiking neurons (Izhikevich \& Edelman 2008). The model contains pyramidal and spiny stellate excitatory cells, together with basket and non-basket interneurons. The modeled neuronal circuit is depicted in Figure 1A. All neuronal types are connected to each other, with the exception 
of supragranular interneurons to layer IV interneurons. Our model contains approximately 2.2 million synapses. The probability of connections and delays in the connections are specific to the types of neurons and based on the experimental literature. The excitatory synapses have specific profiles for their spike-pairing dependent plasticity in accordance with the available literature, as illustrated in Figure 1B. Therefore, our model has approximately 1.8 million dynamical variables. Our simulations were carried out on the Imperial College HPC.

\subsection{Dynamics and response of the model to inputs}

The model accounts for the dynamical activity in the neuronal population (Section 2.5) and we use it to ask whether sensory stimulation affects the spontaneous dynamics of the network. Specifically, can sensory stimulation, via STDP, induce sustained modification in the underlying network weights? Does the network create a dynamical memory of the presented sensory inputs?

Figure 2A shows the average network firing rate over the whole duration of the simulation. The average firing rate drops considerably at the beginning of the simulation until it reaches equilibration at approximately 30 seconds of simulated realtime. The sensory inputs are presented to the network every 10 seconds, starting at simulated realtime 40 seconds (i.e., 10 seconds after the network has reached equilibration) until 70 seconds, and have a 5-second duration (Figure 2B, upper panel). This specific protocol was chosen to allow a relaxation time of the same duration as the duration of the stimulus.

Each 5-second long stimulus block contains 50 repetitions (10 repetitions per second) of the same pattern (Figure 2B, middle panel). This pattern has two parts: a random first half followed by a second half which is the specular image of the first (Figure 2B, lower panel, light and dark grey). This 'back-and-forth' pattern approximates the protraction-retraction motion of the whiskers when rodents explore their environment. The patterns of activity of each neuronal type are shown in Figure $2 \mathrm{C}$ as raster plots. The spike times of a representative proportion of each neuronal type and of all the thalamic (VPm) inputs to the network (colour coding as in Figure 1) are shown for a few milliseconds prior and following the start of the sensory input block at 60 seconds. The main drivers of the network dynamics are the recipients of the thalamic input (i.e., the spiny stellates, $s s_{I V}$, and the basket, $b_{I V}$ and non-basket, $n b_{I V}$ interneurons). The activity spreads from those to the neurons in layers II and III. To aid the visualisation of the network firing during spontaneous and sensory-driven regimes, a movie can be found in the Supplementary Material.

\subsection{Network attractor state modification induced by sensory stimulation}

The dynamics of this complex neuronal network is described by its internal variables, i.e., the synaptic weight variables. As explained in Section 2, the parameters and internal noise sources were chosen to guarantee the stability of the network, its compatibility with externally observed firing rates as reported in the literature, as well as being based on published physiological parameters. Given these constraints and the types of inputs, the dynamics of the network is restricted to a particular 


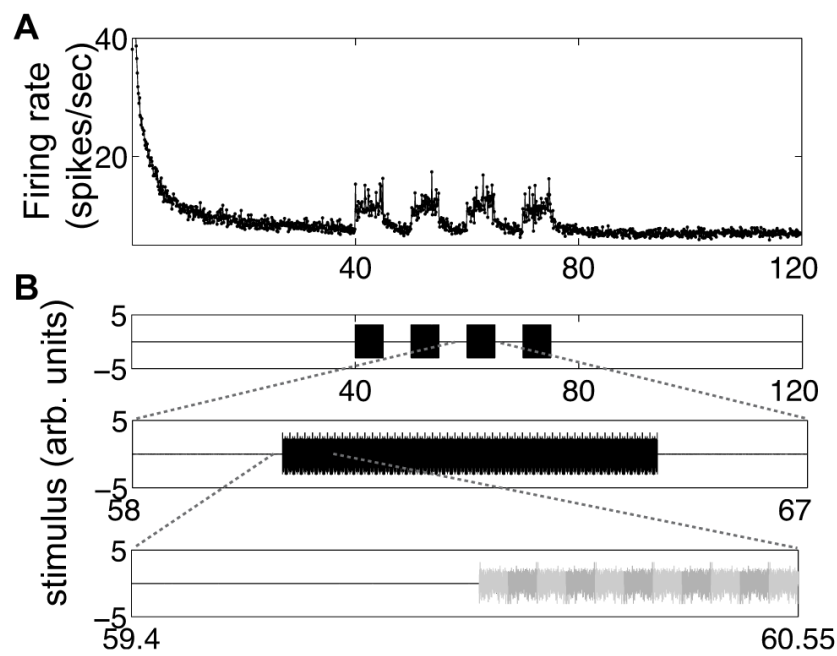

C

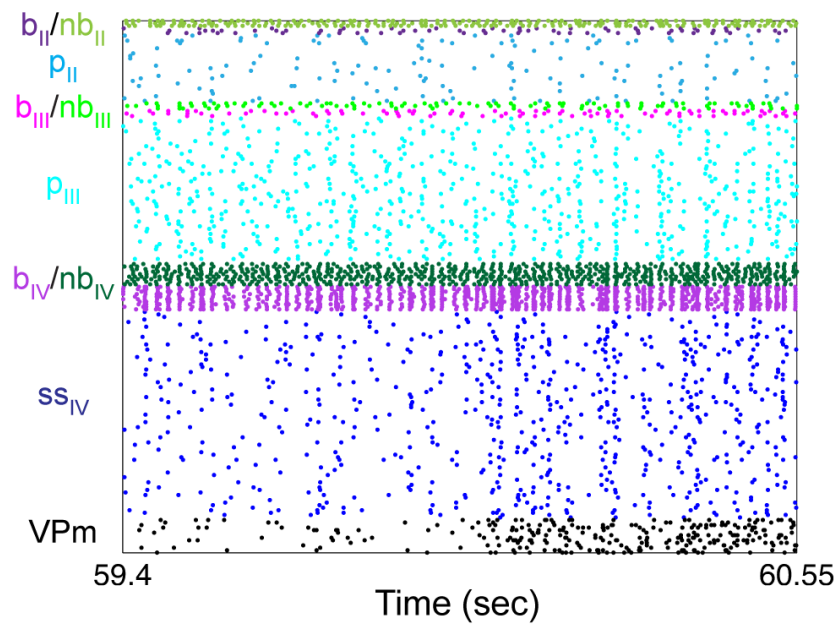

Fig. 2 The stimulation protocol and the response of the model to the stimulus. (a) The average firing rate of the network drops considerably at the beginning of the simulation until it reaches equilibration at approximately 30 seconds of simulated realtime. In order to maintain neuronal activity at constant levels, miniature excitatory potentials were introduced at all synapses as Poisson noise and the weights of the synapses were adjusted to produce firing rates close to those recorded in vivo. The STDP rules introduced in the excitatory synapses allow for network plasticity. The 5-second stimulus is presented 4 times: every 10 seconds from time 40 to 70 seconds. The stimulus produces increased firing rates in the network but the firing rates return to baseline quickly when the stimulus ceases. (b) Each of the four repetitions of the stimulus (upper panel) consists of 10 repetitions of a back-and-forth pattern of white noise: two blocks of white noise, one being the mirror image of the other, in order to mimic the protraction-retraction motion of the whiskers during exploration (light and dark grey, lower panel). The pattern also mimics the $10 \mathrm{~Hz}$ vibrations of the whiskers when exploring the environment. (c) The spiking patterns for each neuronal type (color-coding as in Figure 1) are shown in the raster plot. The spike times are taken from a representative proportion of each neuronal type and from all thalamic (VPm) inputs to the network for a few milliseconds prior and following the start of the sensory input at 60 seconds. 
region of the high-dimensional dynamical space, which we aim to capture through dimensionality reduction.

The average synaptic weight of the coarse-grained system is shown in Figure 3A and the epochs which correspond to the sensory inputs are marked in grey. We wish to investigate whether the pre-stimulus (Figure 3A, dark red) and post-stimulus states of the system (Figure 3A, bright red) are different following consecutive repetitions of the same stimulus. As shown by Figure 3A, there are no differences purely based on the average weights - this is too coarse a measure. Similarly, the firing rates return to baseline after the end of the stimulation protocol and show no significant differences following the epochs (Figure 2A).

The differences induced by the stimulation protocol can however be detected through the analysis of the full dynamical state vector of the network $W(t)=$ $\left[w_{1}(t), w_{2}(t), \ldots, w_{M}(t)\right]$, where the $w_{i}(t)$ are the weights of $\mathrm{M}$ individual synapses $i$. In order to disentangle the complex dynamics of such a large and complex network with very many coupled elements, we opted to study the time evolution of its state space trajectories in a reduced space obtained as follows. First, we obtain a coarse-grained representation of the dynamics, ensuring that we maintain the initial proportions of each neuronal type in the original model: from the approximately $1,700,000$ excitatory synapses in the original model, we reduce our state vector to 2983 by averaging over blocks of 600 weights.

To allow visualisation of this high-dimensional state vector, we projected onto a reduced dimensional space using MDS with the s-stress criterion for minimization of the error between the original and the projected space distances (see Section 4). We applied MDS up to 12 dimensions and plotted the corresponding stress-dimension plot (Figure 3B). An 'elbow' appeared at dimension 2 and the stress decreased very slowly thereafter, indicating a good dimensionality reduction for $D=2$ or $D=3$ (Kruskal \& Wish 1978). We therefore decided to project the state vector onto 3 dimensions for ease of visualisation.

Each point in the resulting plot (Figure 3C) gives a 3-dimensional representation of the synaptic weight of the whole neuronal ensemble at a given time. Figure 3 shows how, after equilibration, the state vector of the network settles onto a pre-stimulus spontaneous attractor region in the 3-dimensional reduced space (Figure 3C, dark red), with small fluctuations about the attractor due to network intrinsic noise.

We hypothesised that "training" the network with repeated sensory inputs could induce long-term, sustained changes in the synaptic weights that would translate into a modified post-evoked spontaneous state for the system. The fundamental mechanism underlying this modification of the spontaneous state of the network is the synaptic plasticity introduced in our model through the STDP profiles. In general, the synaptic weights increase (decrease) between pre- and post-synaptic neurons if they fire at closely correlated (uncorrelated) times. In turn, increased (decreased) synaptic weights increase (decrease) the probability with which a pre-synaptic neuron induces a spike in the post-synaptic one.

We followed the system state trajectory during the time epochs (color coded in Figure 3A). The first sensory input (Figure 3C, evoked-1, grey) induces a substantial deviation of the spontaneous state, causing the neuronal ensemble to diverge from the initial spontaneous attractor. Each of the subsequent sensory inputs (Figure 3C, evoked-2 and evoked-3, grey) caused the ensuing spontaneous trajectories (Figure 3C, post-2 and post-3, brightening red) to progressively deviate from the 
pre-stimulus spontaneous state attractor. Eventually, the sensory-evoked states appear closer in space indicating that the system has adapted to the input. After four instances of external sensory inputs, the spontaneous state attractor is shifted from its initial pre-stimulus state to a new post-stimulus spontaneous state (Figure 3C, bright red). The post-stimulus spontaneous state remains stable until the end of the simulation ( 45 seconds later) and represents the final state of the network following repetitive sensory stimulation of the same input (see also movie in Supplementary Material).

5.3 The modification of the spontaneous state is specific to the stimulus presented

The repetition of sensory inputs (Figure 3, grey) presented to the network caused the successive post-stimulus states (Figure 3, shades of brightening red) to move further away from its initial state (Figure 3, pre-stimulus attractor, dark red). In order to assess if the network is trained through repetitive application of the same stimulus to encode the structure of the stimulus in the weights of the network, we examined if the new, post-stimulus spontaneous state can affect the future responses of the network to stimuli. If the post-stimulus state encodes significant features of the sensory input presented so far (i.e., it represents the formation of a memory specific to the previous inputs), then a 'previously unseen' input of random structure should cause no further shifts in the post-stimulus state. On the other hand, another presentation of the same structured input should cause further learning in the network and the post-stimulus spontaneous state to be shifted consistently further away from its initial state.

We compared the response of the network to the presentation of a fifth repetition of the same stimulus to the response to an ensemble of random shuffles. The fifth sensory input ('test') was presented at the time when the trajectory of the network had reached the post-stimulus spontaneous state, i.e., $\sim 80$ seconds of simulated realtime (Figure 3A, marked 'S') and we recorded the post-test spontaneous activity for a further 15 seconds. The test sensory input (Figure 4A, black squares) caused the post-test spontaneous trajectory (Figure 4B, orange squares) to move further away from its preceding state and hence, even further away from its initial pre-stimulus attractor in a manner consistent with the previous training.

We next presented the network with a shuffled stimulus of the same duration (5 seconds) and repeated the procedure another 18 times always starting from the same initial point marked ' $\mathrm{S}$ ' in Figure 4A-B. The shuffled stimuli induced excitability and caused the trajectories to move away from the stable attractor (Figure 4B, evoked trajectories, grey dots), but the post-shuffled spontaneous trajectories (Figure 4B, brown dots) returned to their baseline state.

These results suggest that repetitive applications of the same stimulus, train the network to encode features of the stimulus in the underlying weights. Repetitive applications of the structured stimulus shifts the pre-stimulus synaptic state into a new state that is distinct to that induced by randomly shuffled inputs.

To study whether the trajectory induced by the test input can be predicted by chance, we tested whether it belongs to the distribution described by the shuffled inputs by applying Gaussian Process Regression (GPR) (Rasmussen \& Nickisch 2010). The estimated GP for the shuffled trajectories is fully specified by a mean vector and a kernel matrix (see Section 4). The probabilistic nature of 

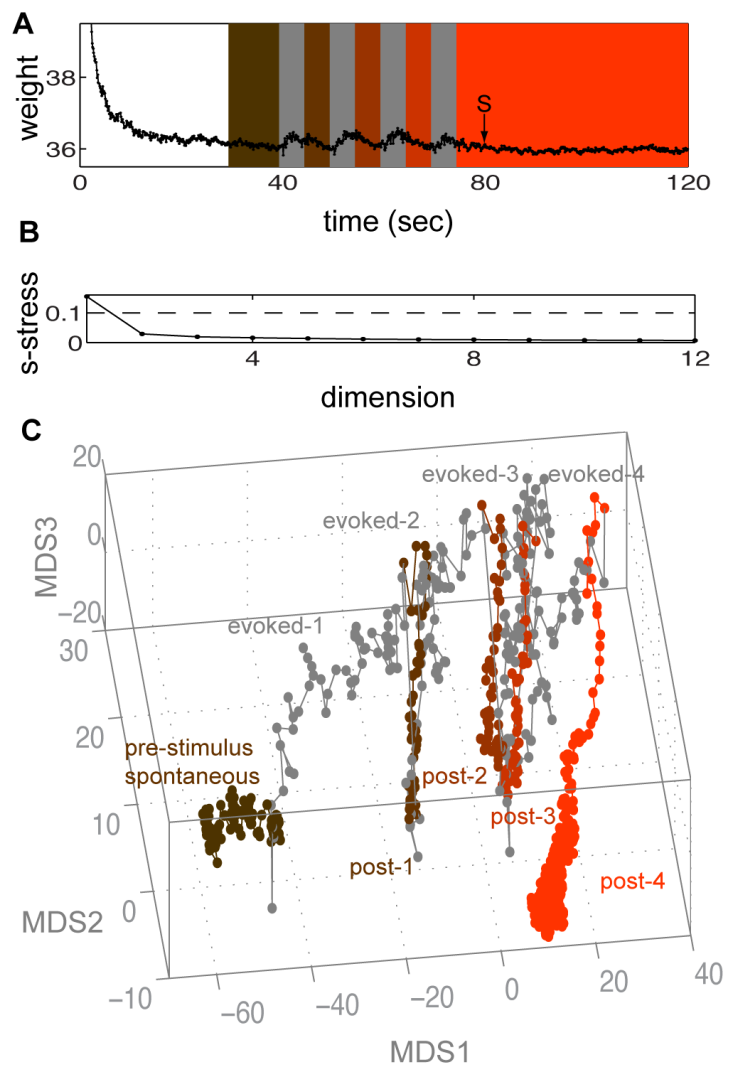

Fig. 3 The pre-stimulus spontaneous attractor state is modified by repetitive sensory stimulation. (a) STDP allows plasticity of the synaptic weights. Stimulation arriving at 40,50,60 and 70 seconds (marked in grey) induces modifications of the average synaptic weight. The pre-stimulus spontaneous state after the network reached equilibration is marked with dark red and each inter-stimulus spontaneous epoch is represented by progressively brighter shades of red. The final post-stimulus spontaneous state of the system is represented by bright red. (b) An 'elbow' in the s-stress plot at dimension $D=2$ indicates that a good dimensionality reduction occurs for all $D=2$ or $D=3$. (c) The time evolution of the state vector of the network on the 3-dimensional reduced space (dark red). The first sensory input (evoked-1, grey) induces a substantial deviation of the spontaneous trajectory causing the neuronal ensemble to diverge from the stable spontaneous attractor. Each of the subsequent sensory inputs (evoked-2 to evoked-4, grey) cause further deviations from the pre-stimulus spontaneous state attractor indicating that the system adapts to the input. After four external inputs the spontaneous state attractor is shifted from its initial stable state to a post-stimulus spontaneous state (bright red). 
GPR provides us with a framework to compute the likelihood of the test trajectory, $s^{*}$ over times $t^{*}$ given the shuffled data set, $\mathbf{s}$ i.e. $p\left(s^{*} \mid t^{*}, \mathbf{s}\right)$ and at each time step, $p\left(s^{*}(i) \mid t^{*}(i), \mathbf{s}\right)$.

As a first step, we identified the axes of maximum variance in the trajectories. To do so, we performed principal component analysis (PCA) on the MDS trajectories induced by the shuffled and test inputs. The axes of maximum variance identified by PCA are shown in Figure 4B (PC1 and PC2, top right). When considered in terms of the time-evolution of the network, the first principal component (PC1) can be seen to correspond to the dynamics of excitation from spontaneous (Figure 4B, red/orange markers) to evoked (Figure 4B, grey/black markers) and vice-versa. In other words, the first principal component corresponds to the 'excitability' of the network. The second principal component (PC2) reflects the shift of the spontaneous state following the series of repeated sensory-evoked stimulations. Hence, the second principal component corresponds to the 'sensory-induced learning' or 'network training' that occurs in the system.

Since both the test and shuffled inputs increase the neuronal activity in the network, we expect only small differences in the trajectories along the 'excitability' axis, as seen in the plot of the excitability of the sensory-evoked and post-stimulus spontaneous trajectories (from 80 to 100 seconds corresponding to the 5 -second stimulation and 15-second post-stimulus spontaneous activity) for both the test and shuffled inputs (Figure 5A, upper panel). However, the dynamics along the 'training' axis shows that the post-test spontaneous trajectory (Figure 5A, lower panel, orange squares) follows a different path when compared to the post-shuffled spontaneous cloud (Figure 5A, lower panel, brown).

The above observations can be quantified calculating the likelihood with which the post-test trajectory can be predicted by the post-shuffled trajectories at every time point. As explained in Section 4.2, we used 19 out of the 20 trajectories generated by the shuffled inputs to estimate the $G P_{\text {shuffled }}$, which we cross-validated against the $20^{\text {th }}$ shuffled input trajectory. The $G P_{\text {shuffled }}$ can be used to calculate the mean (Figure 5A, lower panel, black line) and the $95 \%$ confidence interval (Figure 5A, lower panel, dotted lines) of a representative shuffled trajectory from 80 to 100 seconds.

This process can be used to estimate the likelihood with which each time point of the $20^{\text {th }}$ shuffled input and the test structured input (both new to the GP estimator) can be predicted by this $\mathrm{GP}_{\text {shuffled }}$. We found that the post-test spontaneous trajectory (Figure 5B, upper panel, orange squares) is distinguishable from the cloud of shuffled trajectories: the likelihood with which the test values are predicted by the $\mathrm{GP}_{\text {shuffled }}$ is very small when compared to the likelihood with which the $20^{\text {th }}$ post-shuffled spontaneous trajectory values can be predicted (Figure 5B, upper panel, brown). It is worth noting that the evoked trajectories of the test and shuffled inputs only significantly separate towards the end of the stimulus presentation, at approximately 84 seconds. At this time point and for the remaining 1 second of stimulation time, the likelihood of the evoked-test trajectory targets (Figure $5 \mathrm{~B}$, black squares) to be predicted by the shuffled $\mathrm{GP}_{\text {shuffled }}$ remains at low values. In order to compare the likelihoods of shuffled and structured trajectories, we calculated the ratio of the likelihoods of the structured to the shuffled trajectory. If the two trajectories belonged to the same Gaussian process, their likelihood ratio at all times should be close to 1 . We found that the structured:shuffled likelihood ratio does not differ from 1 at all time points for 

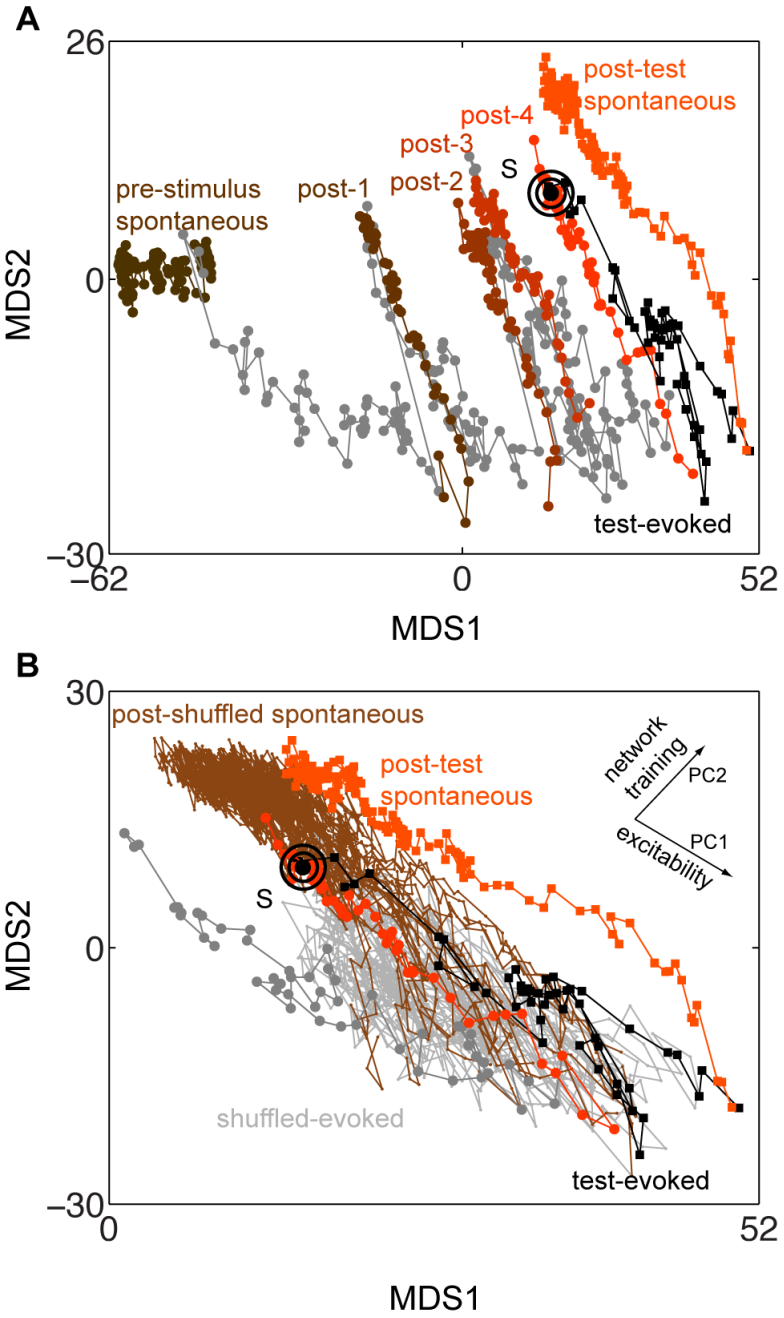

Fig. 4 The network attractor state modification is unique to the stimulus presented. (a) A new repetition of the same input (test, black squares) presented to the system at the post-stimulus spontaneous state (starting point ' $\mathrm{S}$ ' corresponding to time 80 seconds) caused the post-test spontaneous trajectory (orange squares) to move further away from its preceding state. (b) The shuffled stimuli evoked trajectories (grey dots) caused no learning in the network since the post-shuffled spontaneous trajectories (brown dots) return to their baseline state. The application of PCA to the evoked-shuffled, post-shuffled spontaneous, test and post-test spontaneous trajectories identified two axes of maximum variance ( $\mathrm{PC} 1$ and $\mathrm{PC} 2$, top right). Analysed in terms of the time-evolution of the network, the first principal component (PC1) corresponds to the 'excitability' of the network, i.e., the evolution of the network from spontaneous to evoked states and vice-versa. The second principal component (PC2) corresponds to 'network training', i.e., the shift of the spontaneous states as a result of the repeated application of the same sensory stimulation. 
the first 4 seconds of the stimulus presentation. However, towards the end of the stimulus presentation (at approximately 84 seconds) the likelihood ratio drops to zero and remains at values smaller than 1 until the end of the spontaneous epoch at 120 seconds of simulated realtime (Figure 5B, lower panel).

In conclusion, repetitive presentation of a structured input to a neuronal network of layers II, III and IV of a barrel cortical column causes training in the network. STDP present at the excitatory synapses allows the synaptic weights to be modified by stimulation and the network to be trained to the specific features of the structured stimulus. The state of the weights of the network shifts to a poststimulus spontaneous state, which differs significantly from a random modification. In contrast with the repeated exposition to the structured stimulus, presentation of shuffled inputs to the network cause no training and the post-shuffled spontaneous trajectories return to baseline. Thus, repetitive application of structured stimuli gives rise to a post-stimulus state in the weight space, which is unique to the stimulus presented.

\section{Discussion}

In this study, we report the development of a large-scale physiologically-inspired model of barrel cortical layers II, III and IV. Our model incorporates a large amount of published experimental findings on the barrel cortical column, from the types of neurons present and the types of STDP in the excitatory synapses to the probabilities of connections and delays of action potential transmission between neurons. We use this computational model to study the interplay between sensory experience and ongoing spontaneous activity.

6.1 Interplay between spontaneous and sensory-evoked states

At rest, the spontaneous activity converges to a stable state in our model and it is susceptible to learning via sensory experience. Repetitive exposure to the stimuli cause progressive adaptation in the network with the evoked states appearing closer together in the synaptic space and the learned spontaneous state having a stronger dynamical link to the evoked states. In agreement with these results, a recent study in a neural network model reports that in the absence of an input, the neural dynamics itinerate over several states corresponding to each of the memorised patterns after many targets have been learned (Kurikawa \& Kaneko 2011).

Additionally, each time our network is exposed to sensory experience, the subsequent spontaneous synaptic state shifts further away from the initial pre-stimulus attractor. The fact that the sensory-evoked states appear closer in the projected space suggests that the network is trained and progressively learns features of the detailed structure of the stimulus. Learning manifests in the shift of the spontaneous synaptic state of the system. Consecutive repetitions of the same external sensory input induce long-term modifications of the synaptic weights and a new 'learned' (post-stimulus) spontaneous state is created. The modulation of the system occurs through the physiological 'Hebbian' spike-pairing synaptic evolution, 
A
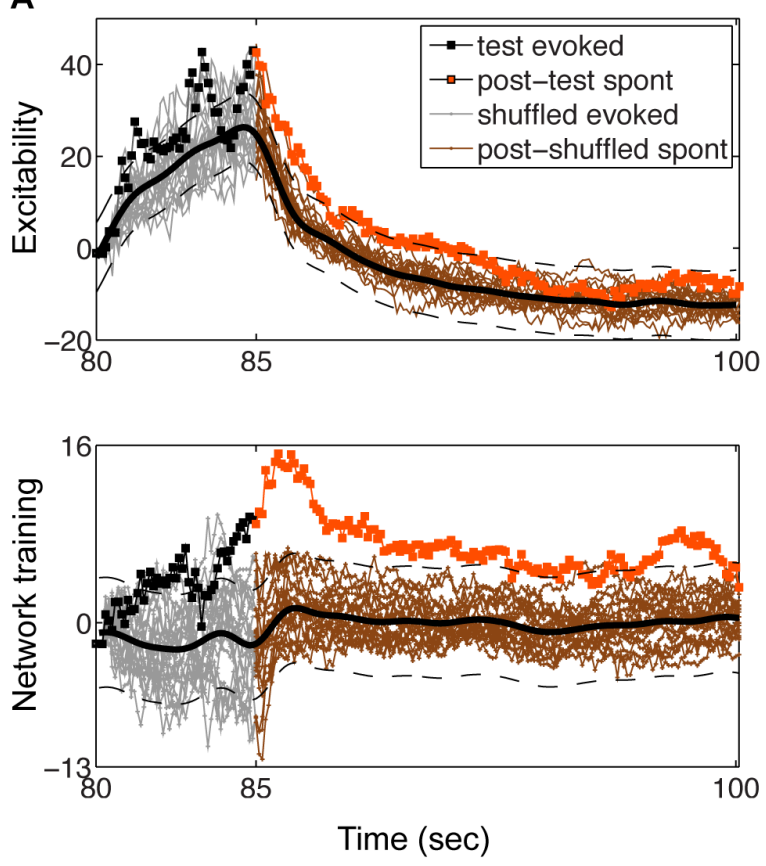

B
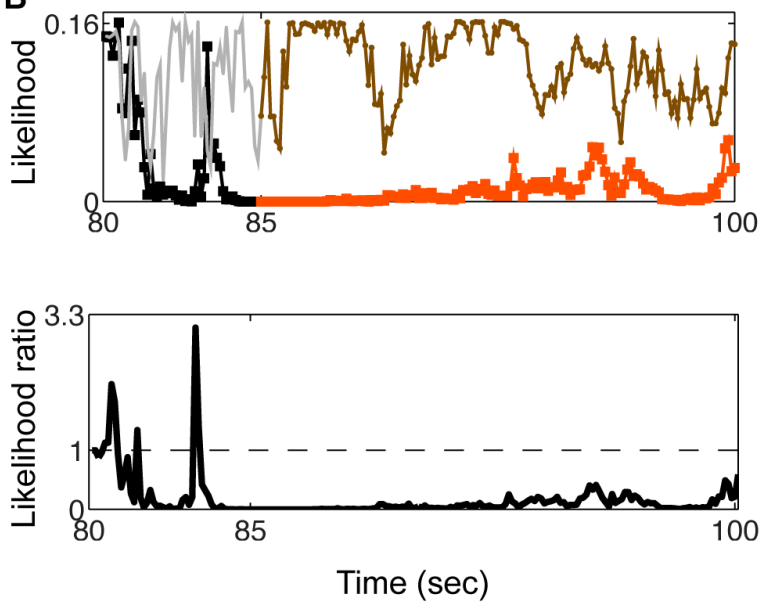

Fig. 5 The structured input induced trajectory is distinguishable from a random response. (a) (Top panel) The 'excitability' of the network (PC1, defined in Figure 4B) during a new sensory input (sensory-evoked and post-stimulus spontaneous trajectories from 80 to 100 seconds) is indistinguishable for a new repetition of the structured input (test, squares) and for an ensemble of shuffled inputs (lines). (Bottom panel) The 'network training' (PC2, defined in Figure 4B) for the structured test and shuffled inputs shows significant differences. The post-test spontaneous trajectory (orange squares) follows a different path to the post-shuffled spontaneous cloud (brown points). The evoked test- (black squares) and shuffled- (light grey points) induced trajectories only significantly separate towards the end of the stimulus presentation at approximately 84 seconds. A representative trajectory from 80 to 100 seconds is predicted by the $\mathrm{GP}_{\text {shuffled }}$ with a mean (black line) and $95 \%$ confidence interval (black dotted line). (b) The trajectory induced by the test stimulus can be predicted by $\mathrm{GP}_{\text {shuffled }}$ with very small likelihood for most of the time (black-red squares). Meanwhile, a different (unused) input from the same shuffled ensemble can be predicted with high likelihood (grey-brown line). The likelihood ratio between the structured and shuffled trajectories is consistently below 1 indicating that the test trajectory is significantly different to the cloud of shuffled trajectories, specifically after approximately 84 seconds until the end of the simulation. 
which modifies the strength of the connection between pre- and post-synaptic neurons depending on whether they fire at closely correlated times. Therefore, spikepairing could be a possible mechanism for long-term sensory-induced modification of the spontaneous cortical dynamics. Our results indicate that physiological STDP profiles present in the excitatory synapses are enough to induce unsupervised longterm synaptic modifications induced by sensory experience. The stimulus induces, via STDP, long-lasting modification in the synaptic weights.

\subsection{Spontaneous state of the network}

The average spontaneous firing rates of the network increase during sensory experience but return to baseline levels thereafter, indicating that the average firing rates may be an inadequate description of the effect of sensory experience on the spontaneous activity in our model. The neuronal firing rates in our model were set by adjusting the synaptic release and weights at the synapses. Possibly because of the symmetric STDP profile at layer IV synapses, the neuronal firing rates in this layer were constrained to values below the physiological norm. Further work needs to shed light on the mechanism, which keeps layer IV synapses stable and neuronal firing rates at higher values. Although this limitation, our model captures the synaptic modifications induced by sensory experience. We show that sensory experience reverberates into the following spontaneous state dynamics via modification of the synaptic weights of the network. In the absence of sensory experience, the synaptic vector converges to an initial spontaneous state. Spontaneous activity has been extensively studied in the framework of attractor neural networks, and it is reported that a prerequisite for stable spontaneous activity is the interplay between excitation and inhibition (since the spontaneous activity is unstable when all neurons are excitatory) (Amit \& Brunel 1997). Here, we report stable spontaneous activity in a complex network of $\sim 4000$ barrel cortical neurons in the presence of constant background noise, modelled as 'minis' in the network.

Our findings suggest that the state of the brain in the absence of sensory stimulation could contribute to its response to subsequent sensory experience. It was previously shown that the state of the brain at the time of the stimulation influences the activity of visual cortex during stimulation (Arieli et al. 1996, Ringach 2003). Would sensory inputs presented to the pre-stimulus state produce the same trajectory as the ones presented to the 'learned' state? Here, we presented the 'learned' state of the network with two types of stimuli: another repetition of the structured stimulus presented before and 'unseen' shuffled stimuli. When the shuffled stimuli are presented, the network is excited by the stimulation but the post-shuffled spontaneous trajectories return to the baseline 'learned' state, indicating that these inputs do not induce further learning in the state of the system. On the other hand, when the previously used stimulus is presented again, the trajectory of the network further deviates from its baseline state. As a result, sensory experience reverberates into the succeeding spontaneous state of the system, and repetitive sensory presentation of the same stimulus shifts the attractor of the system. These results suggest a functional role for spontaneous activity. In agreement with a study of spontaneous activity in a probabilistic Bayesian framework (Berkes et al. 2011), the spontaneous activity of the brain can be seen as the prior upon which future responses are dependent. In this sense, the network favours sensory inputs it has 
already 'seen', suggesting that the learned spontaneous state contains features of the sensory experience encoded in the underlying weights of the network.

\subsection{Comparison with other models}

Our model is able to distinguish between structured and shuffled stimuli possibly because it encodes the spatiotemporal structure of the stimuli in the underlying (internal) state of the network. Simple models, such as the perceptron and multi-layer perceptron are able to classify complex spatial patterns but unlike our model, there is no representation of time (Buonomano \& Maass 2009). Spiking models of the sensory cortex account for the ability of cortical neurons to develop spatial properties such as retinotopy, somatotopy and orientation selectivity, but they don't account for the spatiotemporal structure of natural stimuli (Song \& Abott 2001, Somers et al. 1995, Buonomano \& Maass 2009). Unlike these initial models, our large-scale model incorporates time by including spiking-model units and spike-pairing plasticity in the excitatory synapses. At each time point the response of the neuronal population is dependent not only on the synapses that are directly activated by the input, but also on the ongoing activity in the network (Buonomano \& Maass 2009). As a result, the evolution of the network trajectory with time depends both on the active units and also on the time-dependent plasticity rules present in the synapses. Thus, for a neuronal population to be able to distinguish between spatiotemporal rich stimuli, both of these should be present.

The spike-pairing introduced at the synapses is part of the internal properties of the network and thus, are important for decoding stimuli. An advantage of our model, over other models, is the use of different STDP rules in different synapses, such that the learning temporal windows and symmetry of the STDP profiles differ from synapse-to-synapse. Most of the available spiking neuron models with STDP assume a default symmetric profile at all synapses with the same learning windows (e.g. Kitano \& Fukai 2004, Rao \& Sejnowski 2001, Van Rosssum et al. 2000). Given that STDP learning rules can modify sensory processing, for example modify receptive fields and orientation preferences in adult cats (Yao \& Dan 2001, Fu et al. 2002), it is likely that the temporal window of learning plays an important role.

Other forms of plasticity such as short-term plasticity were excluded from our model. Short-term synaptic plasticity can provide a memory of the recent stimulus history of the network and may continue to change the internal state of the system following stimulus cessation for hundreds of milliseconds (Buonomano \& Maass 2009). Thus, the internal state of the network determined by both the ongoing activity and time-dependent changes in synaptic and cellular state (Buonomano \& Maass 2009) is likely to be dependent on the form of time-dependent neuronal properties present. STDP accounts for the long-term modifications observed in the synapses in our model; whether short-term plasticity will converge or diverge the trajectories induced by the structured or shuffled stimuli remains to be examined.

Acknowledgements We would like to thank Rasmus Petersen for kindly providing us with the thalamic data and Borislav Vangelov for his help with the implementation of the Gaussian Process Regression analysis. This work was supported by a BBSRC DTA studentship (EP), EPSRC grant EP/E002331/1 (SRS), EPSRC grant EP/E049451/1 (MB) and by the EPSRC Support Fund. 


\section{References}

Alenda, A., Molano-Mazón, M., Panzeri, S. \& Maravall, M. (2010), 'Sensory input drives multiple intracellular information streams in somatosensory cortex.', J Neurosci 30, 1087210884.

Amit, D. \& Brunel, N. (1997), 'Model of global spontaneous activity and local structured activity during delay periods in the cerebral cortex.', Cereb Cortex 7, 237-252.

Arieli, A., Sterkin, A., Grinvald, A. \& Aertsen, A. (1996), 'Dynamics of ongoing activity: explanation of the large variability in evoked cortical responses.', Science 273, 1868-1871.

Armstrong-James, M. \& Fox, K. (1987), 'Spatiotemporal convergence and divergence in the rat S1 "barrel" cortex.', J. Comp. Neurol. 263, 265-281.

Azouz, R. \& Gray, C. (1999), 'Cellular mechanism contributing to response variability of cotrical neurons in vivo.', J. Neurosci. 19, 2209-2223.

Beierlein, M., Gibson, J. \& Connors, B. (2003), 'Two dynamically distinct inhibitory networks in layer 4 of the neocortex', J. Neurophysiol. 90, 2987-3000.

Berkes, P., Orbán, G., Lengyel, M. \& Fiser, J. (2011), 'Spontaneous cortical activity reveals hallmarks of an optimal internal model of the environment', Science 331, 83-87.

Brecht, M., Grinevich, V., Jin, T., Margrie, T. \& Osten, P. (2006), 'Cellular mechanisms of motor control in the vibrissal system.', Pflugers Arch - Eur J Physiol 453, 269-281.

Bruno, R. \& Simons, D. (2002), 'Feedforward mechanisms of excitatory and inhibitory cortical receptive fields', J. Neurosci. 22, 10966-10975.

Buonomano, D. \& Maass, W. (2009), 'State-dependent computations: spatiotemporal processing in cortical networks.', Nat Rev 10, 113-125.

Carvell, G. \& Simons, D. (1990), 'Biometric analyses of vibrissal tactile discrimination in the rat.', J. Neurosci. 10, 2638-2648.

Chagnac-Amitai, Y., Luhmann, H. \& Prince, D. (1990), 'Burst generating and regular spiking layer 5 pyramidal neurons of rat neocortex have different morphological features', J. Comp. Neurol. 296, 598-613.

Cox, T. \& Cox, M. (2001), Multidimensional Scaling, 2nd edn, Chapman and Hall/CRC.

Creutzfeldt, O., Watanabe, S. \& Lux, H. (1966), 'Relations between eeg phenomena and potentials of single cortical cells. ii. spontaneous and convulsoid activity.', Electroenceph. Clin. Neurophysiol. 20, 19-37.

Cruikshank, S., Urabe, H., Nurmikko, A. \& Connors, B. (2009), 'Pathway-specific feedforward circuits between thalamus and neocortex revealed by selective optical stimulation of axons.', Neuron 62, 413-425.

del Castillo, J. \& Katz, B. (1954), 'Quantal components of the end-plate potential.', J Physiol 124, 560-573.

Egger, V., Feldmeyer, D. \& Sakmann, B. (1999), 'Coincidence detection and changes of synaptic efficacy in spiny stellate neurons in rat barrel cortex.', Nat. Neurosci. 2, 1098-1105.

Feldman, D. (2000), 'Timing-based LTP and LTD at vertical inputs to layer II/III pyramidal cells in rat barrel cortex.', Neuron 27, 45-56.

Feldmeyer, D., Egger, V., Lübke, J. \& Sakmann, B. (1999), 'Reliable synaptic connections between pairs of excitatory layer 4 neurones within a single 'barrel' of developing rat somatosensory cortex.', J. Physiol. 521, 169-190.

Feldmeyer, D., Lübke, J. \& Sakmann, B. (2006), 'Efficacy and connectivity of intracolumnar pairs of layer $2 / 3$ pyramidal cells in the barrel cortex of juvenile rats.', J. Physiol. 575, 583601.

Feldmeyer, D., Lübke, J., Silver, A. \& Sakmann, B. (2002), 'Synaptic connections between layer 4 spiny neurone - layer $2 / 3$ pyramidal cell pair in juvenile rat barrel cortex: physiology and anatomy of interlaminar signalling within a cortical column.', J. Physiol. 538.3, 803-822.

Ferezou, I., Haiss, F., Gentet, L., Aronoff, R., Weber, B. \& Petersen, C. (2007), 'Spatiotemporal dynamics of cortical sensorimotor integration in behaving mice.', Neuron 56, 907-923.

Fiser, J., Chiu, C. \& Weliky, M. (2004), 'Small modulation of ongoing cortical dynamics by sensory input during natural vision.', Nature 431, 573-578.

Fox, M., Snyder, A., Vincent, J. \& Raichle, M. (2007), 'Intrinsic fluctuations within cortical systems account for intertrial variability in human behavior.', Neuron 56, 171-184.

Fu, Y., Djupsund, K., Gao, H., Hayden, B., Shen, K. \& Dan, Y. (2002), 'Temporal specificity in the cortical plasticity of visual space representation.', Science 296, 1999-2003.

Galarreta, M., Erdélyi, F., Szabó, G. \& Hestrin, S. (2008), 'Cannabinoid sensitivity and synaptic properties of 2 GABAergic networks in the neocortex.', Cereb. Cortex 18, 2296-2305. 
Galarreta, M. \& Hestrin, S. (2001), 'Spike transmission and synchrony in networks of GABAergic interneurons.', Science 292, 2295-2299.

Galarreta, M. \& Hestrin, S. (2002), 'Electrical and chemical synapses among parvalbumin fast spiking GABAergic interneurons in adult mouse neocortex.', PNAS 292, 2295-2299.

Gentet, L., Stuart, G. \& Clements, J. (2000), 'Direct measurement of specific membrane capacitance in neurons', Biophys. J. 79, 314-320.

Gewaltig, M. \& Diesmann, M. (2007), 'NEST (NEural Simulation Tool)', Scholarpedia 2, 1430.

Gibson, J., Beierlein, M. \& Connors, B. (1999), 'Two networks of electrically coupled inhibitory neurons in neocortex.', Nature 402, 75-79.

Gibson, J., Beierlein, M. \& Connors, B. (2005), 'Functional properties of electrical synapses between inhibitory interneurons of neocortical layer 4.', J. Neurophysiol. 93, 467-480.

Gütig, R., Aharonov, R., Rotter, S. \& Sompolinsky, H. (2003), 'Learning input correlations through nonlinear temporal assymetric hebbian plasticity.', J. Neurosci. 23, 3697-3714.

Halabisky, B., Shen, F., Huguenard, J. \& Prince, D. (2006), 'Electrophysiological classification of somatostatin-positive interneurons in mouse sensorimotor cortex', J. Neurophysiol. 96, 834-845.

Han, F., Caporale, N. \& Dan, Y. (2008), 'Reverbation of recent visual experience in spontaneous cortical waves.', Neuron 60, 321-327.

Hartline, H. K. (1938), 'The response of single optic nerve fibbers of the vertebrate eye to illumination of the retina', Am J Physiol 121, 400-415.

Hasenstaub, A., Sachdev, R. \& DA., M. (2007), 'State changes rapidly modulate cortical neuronal responsiveness.', J Neurosci 27, 9607-9622.

Helmstaedter, M., Staiger, J., Sakmann, B. \& Feldmeyer, D. (2008), 'Efficient recruitment of layer $2 / 3$ interneurons by layer 4 input in single columns of rat somatosensory cortex.', $J$. Neurosci. 28, 8273-8284.

Holmgren, C., Harkany, T., Svennenfors, B. \& Y., Z. (2003), 'Pyramidal cell communication within local networks in layer $2 / 3$ of rat neocortex.', J. Physiol. 555.1, 139-153.

Hubel, D. \& Wiesel, T. (1968), 'Receptive fields and functional architecture of monkey striate cortex.', J. Physiol. 195, 215-243.

Izhikevich, E. \& Edelman, G. (2008), 'A large-scale model of mammalian thalamocortical systems.', PNAS 105, 3593-3598.

J., S. (1976), 'Organization of spontaneous electrical activity in neocortex.', Prog. Brain Res. 45, 309-325.

Jin, T., Witzemann, V. \& Brecht, M. (2004), 'Fiber types of the intrinsic whisker muscle and whisking behavior.', J Neurosci 24, 3386-3393.

Kawaguchi, Y. (1995), 'Physiological subgroups of nonpyramidal cells with specific morphological characteristics in layer II/III of rat frontal cortex.', J. Neurosci. 15, 2638-2655

Kenet, T., Bibitchkov, D., Tsodyks, M., Grinvald, A. \& Arieli, A. (2003), 'Spontaneously emerging cortical representations of visual attributes.', Nature 425, 954-956.

Killackey, H. \& Ebner, F. (1973), 'Convergent projection of three separate thalamic nuclei on to a single cortical area', Science 179, 283-285.

Kitano, K. \& Fukai, T. (2004), 'Temporal characteristics of the predictive synchronous firing modeled by spike-timing-dependent plasticity.', Learn Mem 11, 267-276.

Kruskal, J. (1964), 'Multidimensional scaling by optimizing goodness of fit to a nonmetric hypothesis', Psychometrica 29, 1-27.

Kruskal, J. \& Wish, M. (1978), Multidimensional Scaling, SAGE Publications.

Kurikawa, T. \& Kaneko, K. (2011), 'Learning shapes spontaneous activity itineating over memorized states.', Plos One 6, e17432-.

Kyriazi, H. \& Simons, D. (1993), 'Thalamocortical response transformations in simulated whisker barrels.', J Neurosci 13, 1601-1615.

Le Bon-Jego, M. \& Yuste, R. (2007), 'Persistently active, pacemaker-like neurons in neocortex.', Front. Neurosci. 1, 123-129.

Lee, K. \& Woolsey, T. (1975), 'A proportional relationship between peripheral innervation density and cortical neuron number in the somatosensory system of the mouse.', Brain Res. 99, 349-353.

Lefort, S., Tomm, C., F.J.C., S. \& Petersen, C. (2009), 'The excitatory neuronal network of the C2 barrel column in mouse primary somatosensory cortex', Neuron 61, 301-316.

Lewis, C., Baldassarre, A., Committeri, G., Romani, G. \& Corbetta, M. (2009), 'Learning sculpts the spontaneous activity of the resting human brain.', PNAS 106, 17558-17563.

Lu, J., Li, C., Zhao, J., Poo, M. \& XH., Z. (2007), 'Spike-timing-dependent plasticity of neocortical excitatory synapses on inhibitory interneurons depends on target cell type.', J. Neurosci. 27, 9711-9720. 
Lübke, J., Egge, V., Sakmann, B. \& Feldmeyer, D. (2000), 'Columnar organization of dendrites and axons of single and synaptically coupled excitatory spiny neurons in layer 4 of the rat barrel cortex.', J. Neurosci. 20, 5300-5311.

Luczak, A., Barthó, P. \& Harris, K. (2009), 'Spontaneous events outline the realm of possible sensory responses in neocortical populations', Neuron 62, 413-425.

Luczak, A., Barthó, P., Marguet, S., Buzsáki, G. \& Harris, K. (2007), 'Sequential structure of neocortical spontaneous activity in vivo.', PNAS 104, 347-352.

Mao, B., Hamzei-Sichiani, F., Aronov, D., Froemke, R. \& Yuste, R. (2001), 'Dynamics of spontaneous activity in neocortical slices.', Neuron 32, 883-898.

Margrie, T., Brecht, M. \& Sakmann, B. (2002), 'In vivo, low-resistance, whole-cell recordings from neurons in the anaesthetized and awake mammalian brain.', Pflugers Arch. 444, 491498.

Markram, H., Lübke, J., Frotscher, M. \& Sakmann, B. (1997), 'Regulation of synaptic efficacy by coincidence of postsynaptic APs and EPSPs.', Science 275, 213-215.

Marmarelis, P. \& Naka, K. (1972), 'White-noise analysis of a neuron chain: an application of the Weiner theory', Science 175, 1276-1278.

Mason, A., Nicoll, A. \& Stratford, K. (1991), 'Synaptic transmission between individual neurons of the rat visual cortex in vitro', J. Neurosci. 11, 72-84.

McCormick, D., Connors, B., Lighthall, J. \& Prince, D. (1985), 'Comparative electrophysiology of pyramidal and sparsely spiny neurons of the neocortex.', J. Neurophysiol. 54, 782-806.

Montemuro, M., Panzeri, S., Maravall, M., Alenda, A., Bale, M., Brambilla, M. \& Petersen, R. (2007), 'Role of precise spike timing in coding of dynamic vibrissa stimuli in somatosensory thalamus.', J. Neurophysiol. 98, 1871-1882.

Petersen, C., Hahn, T., Mehta, M., Grinvald, A. \& Sakmann, B. (2003), 'Interaction of sensory responses with spontaneous depolarization in layer $2 / 3$ barrel cortex.', PNAS 100, 1363813643.

Pillow, J. W., Shlens, J., Paninski, L., Sher, A., Litke, A. M., Chichilnisky, E. J. \& Simoncelli, E. P. (2008), 'Spatio-temporal correlations and visual signalling in a complete neuronal population.', Nature 454(7207), 995-9.

Raichle, M. E. \& Mintun, M. A. (2006), 'Brain work and brain imaging.', Annu Rev Neurosci 29, 449-76.

Rao, R. \& Sejnowski, T. (2001), 'Spike-timing-dependent hebbian plasticity as temporal difference learning', Neural Comput 13, 2221-2237.

Rasmussen, C. \& Nickisch, H. (2010), 'Gaussian processes for machine learning (gpml) toolbox', JMLR 11, 3011-3015.

Ringach, D. (2003), 'States of mind', Nature 425, 912-913.

Sakata, S. \& Harris, K. (2009), 'Laminar structure of spontaneous and sensory-evoked population activity in auditory cortex.', Neuron 64, 404-418.

Saleem, A. B., Krapp, H. G. \& Schultz, S. R. (2008), 'Receptive field characterization by spike-triggered independent component analysis.', J Vis 8(13), 2.1-16.

Sokoloff, L., Mangold, R., Wechsler, R. L., Kenney, C. \& Kety, S. S. (1955), 'The effect of mental arithmetic on cerebral circulation and metabolism.', J Clin Invest 34(7, Part 1), 1101-8.

Somers, D., Nelson, S. \& Sur, M. (1995), 'An emergent model of orientation selectivity in cat visual cortical simple cells.', J Neurosci 15, 5448-5465.

Song, S. \& Abott, L. (2001), 'Cortical development and remapping through spike timingdependent plasticity.', Neuron 32, 339-350.

Song, S., Sjorstrom, P., Reigi, M., Nelson, S. \& Chklovskii, D. (2005), 'Highly nonrandom features of synaptic connectivity in local cortical circuits', Plos Biol. 3, 507-519.

Takane, Y., Young, F. \& de Leeuw J. (1977), 'Nonmetric individual differences multidimensional scaling: an alternating least squares method with optimal scaling features.', Psychometrica 42, 7-67.

Thomson, A., West, D., Wang, Y. \& Bannister, A. (2002), 'Synaptic connections and small circuits involving excitatory and inhibitory neurons in layers $2-5$ of adult rat and cat neocortex: triple intracellular recordings and biocytin labelling in vitro', Cereb. Cortex 12, 936-953.

Tsodyks, M., Kenet, T., Grinvald, A. \& Arieli, A. (1999), 'Linking spontaneous activity of single cortical neurons and the underlying functional architecture.', Science 286, 19431946.

Van Rosssum, M., Bi, G. \& Turrigiano, G. (2000), 'Stable Hebbian learning from spike timingdependent plasticity.', J Neurosci 20, 8812-8821. 
Wu, M. C.-K., David, S. V. \& Gallant, J. L. (2006), 'Complete functional characterization of sensory neurons by system identification.', Annu Rev Neurosci 29, 477-505.

Xiang, Z., Huguenard, J. \& Prince, D. (2002), 'Synaptic inhibition of pyramidal cells evoked by different interneuron subtypes in layer V of rat visual cortex.', J. Neurophysiol. 88, 740750 .

Yao, H. \& Dan, Y. (2001), 'Stimulus timing-dependent plasticity in cortical processing of orientation.', Neuron 32, 315-323.

Yao, H., Shi, L., Han, F., Gao, H. \& Dan, Y. (2007), 'Rapid learning in cortical coding of visual scenes.', Nat Neurosci 10, 772-778.

Yuste, R., MacLean, J., Smith, J. \& Lansner, A. (2005), 'The cortex as a central pattern generator.', Nat. Rev. 6, 477-483.

Zhu, J. \& Connors, B. (1999), 'Intrinsic firing patterns and whisker-evoked synaptic responses of neurons in the rat barrel cortex', J. Neurophysiol. 81, 1117-1183. 